\title{
Classification and management of hepatolithiasis: A high-volume, single-center's experience
}

\author{
Xiaobin Feng ${ }^{1}$, Shuguo Zheng ${ }^{1}$, Feng Xia ${ }^{1}$, Kuansheng Ma ${ }^{1}$, Shuguang Wang ${ }^{1}$, Ping Bie ${ }^{1}$, \\ Jiahong Dong ${ }^{1,2, *}$
}

${ }^{1}$ Institute of Hepatobiliary Surgery, Southwest Hospital, Third Military Medical University, Chongqing, China;

${ }^{2}$ Institute of Hepatobiliary Surgery, Chinese PLA General Hospital, Beijing, China.

\begin{abstract}
Summary Hepatolithiasis is endemic to East Asia, but immigration from the region means that this rare but emerging disease will pose a therapeutic challenge to doctors in the West as well. Curative management of hepatolithiasis is difficult since its etiology has not been fully elucidated. Hepatectomy is the best approach to treating hepatolithiasis. Here, we propose a novel classification of hepatolithiasis and describe features of each type. We then relate our experience with various forms of hepatectomy to treat different types of hepatolithiasis. Surgery should be indicated for all cases of hepatolithiasis. The proposed classification will help to determine surgical strategies. Better selection of which patients should undergo a hepatectomy will lead to better outcomes.
\end{abstract}

Keywords: Hepatectomy, hepatolithiasis, classification

\section{Introduction}

Hepatolithiasis is endemic to East Asia, which includes China, South Korea, Japan, the Philippines, Vietnam, Thailand, Malaysia, and Indonesia, and its prevalence there can range as high as $30-50 \%$ (1). This disease involves gallstones in the bile ducts proximal to the confluence of the right and left hepatic ducts, irrespective of the co-existence of gallstones in the common bile duct (CBD) and/or gallbladder (2). In the past, this disease was rare, with a prevalence of $0.6-1.3 \%$ (3) in the West, but it is increasingly encountered in the West because of greater immigration from Asia (4-7).

The etiology of hepatolithiasis has yet to be fully elucidated, although genetic, dietary, and environmental factors are thought to contribute to the disease. Hence, curative management of hepatolithiasis is difficult since treatment depends greatly on fully understanding the mechanism of stone formation. The goal of hepatolithiasis treatment is to resolve ongoing infections, prevent recurrent cholangitis and

*Address correspondence to:

Dr. Jiahong Dong, Institute of Hepatobiliary Surgery, Chinese PLA General Hospital, 28 Fuxing Road, Beijing100853, China.

E-mail: xiaobinf@medmail.com.cn subsequent hepatic fibrosis, decrease the need for recurrent instrumentation, and prevent progression to cholangiocarcinoma (2). Available treatments include medication and surgery. Surgery, including removal of the affected liver segment(s), has been the best treatment thus far. Hepatectomy can remove stones and focal lesions to eliminate the risk of cholangiocarcinoma, strictures, and subsequent bile stasis to provide effective drainage of biliary tract. Complete removal of the diseased lobe or segment is crucial to preventing recurrence and progressive liver disease. The current report describes a system of classifying hepatolithiasis for surgery and firsthand experience managing the disease.

\section{Classification of hepatolithiasis for surgery}

Classifying surgical candidates or indications for surgery is crucial for a hepatectomy to treat hepatolithiasis to result in the best outcome. Normally, the indications for hepatectomy to treat hepatolithiasis are as follows: $i$ ) unilobar hepatolithiasis, particularly left-sided; ii) atrophy, fibrosis, and multiple abscesses secondary to cholangitis; iii) suspicion of concomitant intrahepatic cholangiocarcinoma; and iv) multiple intrahepatic stones with biliary strictures that cannot be treated percutaneously or endoscopically (8). 
The criteria for classification should be based on the pathological characteristics of the biliary tree and hepatic parenchyma. Classification should help to determine treatment strategies and it should be simple and easy to apply. However, there has been no universal classification of hepatolithiasis until now.

Based on previous experience managing hepatolithiasis, the current authors propose a system of classification, designated "Dong's Classification" to determine reasonable surgical approaches to treating hepatolithiasis (Table 1). In this classification, hepatolithiasis is divided into two types, type I and type II. Type I is a localized stone disease with stones located in one (Figure 1A, Type Ia) or both lobes (Figure 1B, Type Ib). Type II is a diffuse stone disease, which is divided into three subtypes: type IIa involves no atrophy of the hepatic parenchyma or stricture of the intrahepatic bile ducts (Figure 2A); type IIb involves segmental atrophy or/and stricture of the intrahepatic bile ducts (Figure 2B); and type IIc involves biliary cirrhosis and portal hypertension (Figure 2C).
The letter "E" represents an additional type of hepatolithiasis with extrahepatic stones. This type is divided into three subtypes: "Ea" representing normal functioning of the sphincter of Oddi; "Eb" representing relaxation of the sphincter of Oddi, and "Ec" representing stricture of the sphincter of Oddi (Figure 3).

\section{Hepatectomy to treat hepatolithiasis}

Data were collected on patients at the Institute of Hepatobiliary Surgery, Southwest Hospital, and Chinese PLA General Hospital, both of which are leading facilities for biliary surgery in China. Hepatectomy to treat hepatolithiasis was initiated at Southwest Hospital by Prof. Zhiqiang Huang in 1957 and was reported by him in a Chinese medical journal in 1959 (9).

Hepatectomy is the best approach to treating hepatolithiasis because it removes stones and also because it removes the strictured bile duct, resects the atrophic portion of the liver, and eliminates the potential presence of cholangiocarcinoma, thus reducing the risk of recurrent stones.

Table 1. Dong's classification of hepatolithiasis for use in determining surgical approaches

\begin{tabular}{cl}
\hline Type & \multicolumn{1}{c}{ Definition or content } \\
\hline Type I & Localized stone disease: unilobar or bilobar. \\
Type II & Diffuse stone disease. \\
IIa & No atrophy of the hepatic parenchyma or stricture of the intrahepatic bile ducts. \\
IIb & Segmental atrophy or/and stricture of the intrahepatic bile ducts. \\
IIc & Biliary cirrhosis and portal hypertension. \\
& \\
Additional Type E & Extrahepatic stones. \\
Ea & Normal sphincter of Oddi. \\
Eb & Relaxation of the sphincter of Oddi. \\
Ec & Stricture of the sphincter of Oddi. \\
\hline
\end{tabular}

A


B


Figure 1. Type I hepatolithiasis. (A), Type Ia. Localized stone disease with stones located in only one lobe. In this case, stones were localized in the atrophic right anterior portion of the liver. Segmentectomy of S5 and S8 was performed; (B), Type Ib. Localized stone disease with stones located in both lobes. In this case, stones were localized in the atrophic right anterior portion and left lateral portion of the liver. 
A

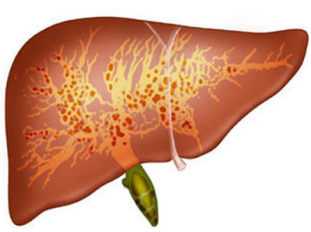

B

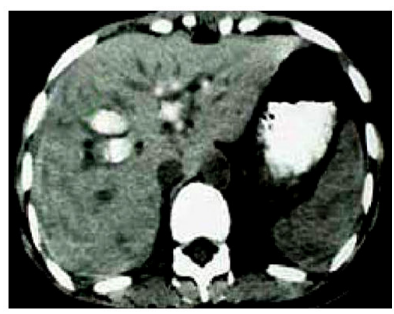



C
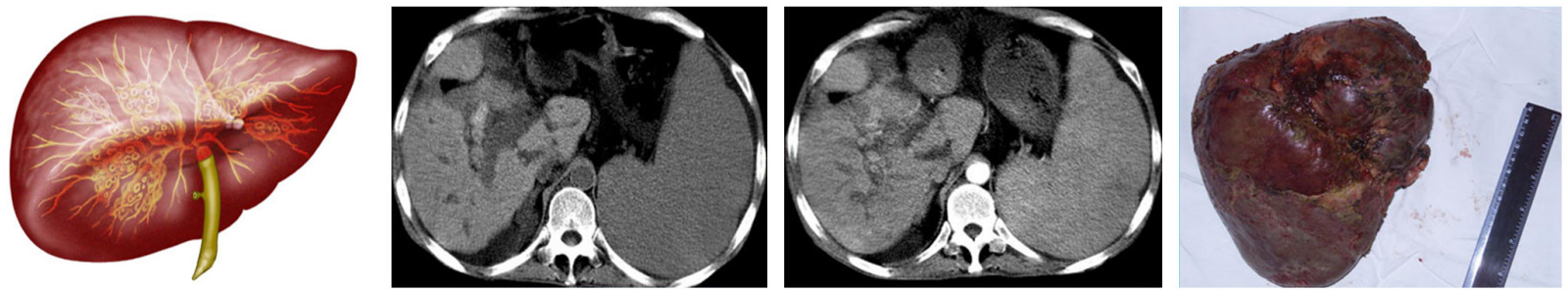

Figure 2. Type II hepatolithiasis. (A), Type IIa. Diffuse stone disease without segmental atrophy or stricture; (B), Type IIb. Diffuse stone disease with segmental atrophy and/or stricture. In this case, stones were located in every liver segment and there was atrophy or biliary stricture of S2, S3, S6, and S7; (C), Type IIc. Diffuse stone disease with secondary biliary cirrhosis. In this case, stones were located in every liver segment and there was biliary cirrhosis and portal hypertension. A liver transplant is needed.

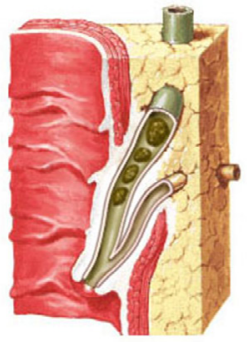

Ea: normal



Eb: relaxation

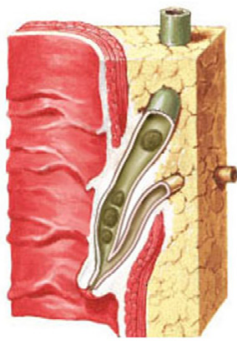

Ec : stricture
Figure 3. Additional type E hepatolithiasis. Hepatolithiasis with extrahepatic stones. Hepatolithiasis with extrahepatic stones (E) is divided into three subtypes based on functioning of the sphincter of Oddi. Normal function of the sphincter of Oddi is designated "Ea", relaxation of the sphincter of Oddi is designated "Eb", and stricture of the sphincter of Oddi is designated "Ec".

\subsection{Indications for hepatectomy to treat hepatolithiasis}

Based on experience, patients with type I and type IIb hepatolithiasis are the best candidates for hepatectomy. In type I localized stone disease, surgery resects the stone-bearing segments, regardless of where atrophy or a stricture is found (Figure 4). Type II involves a high risk of stone recurrence, so all patients with type II should undergo stone removal along with a Rouxen-Y hepaticojejunostomy $(10,11)$ or hepaticocutaneous jejunostomy (12-15). Hepatectomy is the best way to resect lesions in patients with type IIb hepatolithiasis and hepatic lesions (e.g. segmental atrophy hepatic abscess or cholangiocarcinoma). Patients with type IIc hepatolithiasis consistently have biliary cirrhosis, portal hypertension, and liver failure as well, indicating the need for a liver transplant. Additionally, a hepaticojejunostomy should also be performed to treat "Eb" and "Ec" hepatolithiasis.

\subsection{Outcomes of hepatolithiasis treated by surgery}

From June 1976 to June 2009, 1,930 patients underwent surgery for hepatolithiasis at the Institute of Hepatobiliary Surgery. Of these, 1,175 patients underwent hepatectomy and 755 patients primarily underwent liver-preserving surgery and stone removal.

Perioperative outcomes showed that patients who underwent a hepatectomy had much greater intraoperative bleeding than patients who had stones removed. Hepatectomy was associated with a longer operating time than stone removal. There were no significant differences between the patients in terms of mortality and morbidity. However, the postoperative rate of residual stones was much lower after hepatectomy than after stone removal (Table 2).

The operative morbidity rate was $13.3 \%$ for 1,175 patients who underwent a hepatectomy. The most common complication was wound infection, followed by biliary leakage, pleural effusion, pneumonia, stress ulcer, intraabdominal bleeding, and hepatic failure (Table 3).

\subsection{Typical cases where a hepatectomy was used to treat hepatolithiasis}


$\underline{A}$


B
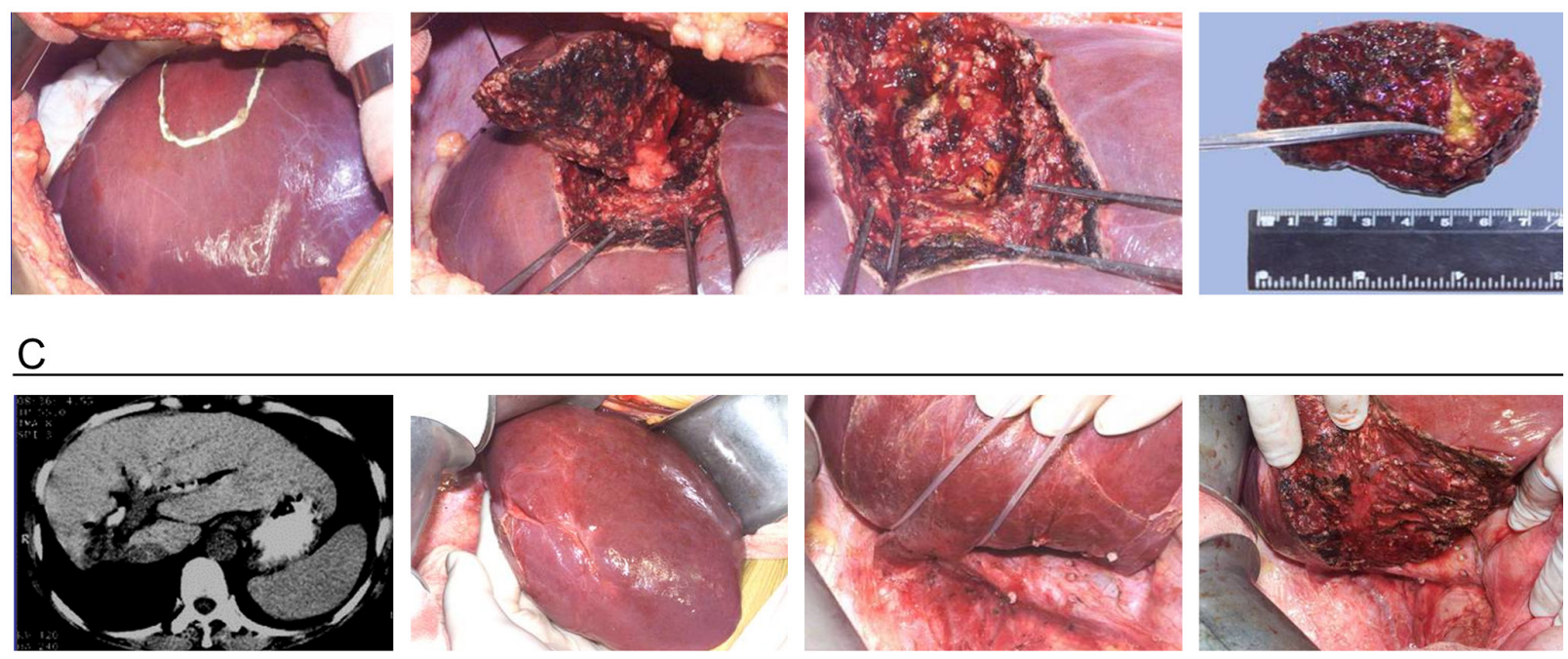

Figure 4. Typical selection of the procedure to treat type I hepatolithiasis. (A), In this case, anatomic left lobectomy was performed to treat type I hepatolithiasis; (B), In this case, segmentectomy of S8 was performed; (C), In this case, a right posterior sectionectomy was performed.

Table 2. Perioperative outcomes for 1,930 patients with hepatolithiasis (June, 1975 - June, 2008)

\begin{tabular}{lccr}
\hline & $\begin{array}{l}\text { HT group } \\
(n=1,175)\end{array}$ & $\begin{array}{c}\text { SR group } \\
(n=755)\end{array}$ & $P$ Value \\
\hline Operating time (min) $^{*}$ & $332 \pm 123$ & $289 \pm 106$ & $<0.001$ \\
Blood loss (mL) $^{*}$ & $717 \pm 712$ & $443 \pm 510$ & $<0.001$ \\
Postoperative mortality $^{\#}$ & $3(2.6 \%)$ & $1(1.3 \%)$ & 1.000 \\
Residual stones $^{\#}$ & $224(19.1 \%)$ & $332(44.0 \%)$ & $<0.001$ \\
Perioperative complications $^{\#}$ & $156(13.3 \%)$ & $93(12.3 \%)$ & 0.157 \\
\hline
\end{tabular}

HT, Hepatectomy; SR, Stone Removal; ;, data are expressed as average \pm S.D.;, , data represent cases (ratio).

Table 3. Postoperative complications in 1,175 patients who underwent a hepatectomy (June, 1975 - June, 2008)

\begin{tabular}{lc}
\hline Types of Complications & Patients (\%) \\
\hline Wound infection & $81(6.9 \%)$ \\
Biliary leakage & $26(2.2 \%)$ \\
Pleural effusion & $21(1.8 \%)$ \\
Pneumonia & $14(1.2 \%)$ \\
Stress ulcer & $13(1.1 \%)$ \\
Abdominal bleeding & $6(0.5 \%)$ \\
Hepatic failure & $3(0.3 \%)$ \\
\hline
\end{tabular}

\subsubsection{Anatomic left lobectomy, segmentectomy, and} sectionectomy for type I hepatolithiasis

In a case of stones localized in the atrophic left lobe and extrahepatic bile duct, the hepatolithiasis was classified as type I plus Ea, and anatomic left lobectomy was performed and extrahepatic stones were removed (Figure 4A). In a case of a stone localized in atrophic segment VIII, hepatolithiasis was classified as type I, and segmentectomy of S8 was performed (Figure $4 \mathrm{~B})$. In a case of stones localized in the atrophic right posterior portion of the liver, anatomic right posterior sectionectomy was performed (Figure 4C).

\subsubsection{Bilateral lobectomy excluding the caudate lobe for type II hepatolithiasis}

From March 2003 to July 2011, 252 patients with type II hepatolithiasis at this Institute underwent surgery. Of these, $12(4.8 \%)$ underwent bilateral lobectomy excluding the caudate lobe. In a typical case, a 39-yearold female had diffusely distributed stones with atrophy in all segments except for the caudate lobe (Figure 5). All 12 of the patients successfully underwent bilateral lobectomy excluding the caudate lobe.

\section{Conclusion}

Economic development and a more Western lifestyle are associated with a decline in the incidence of 
A
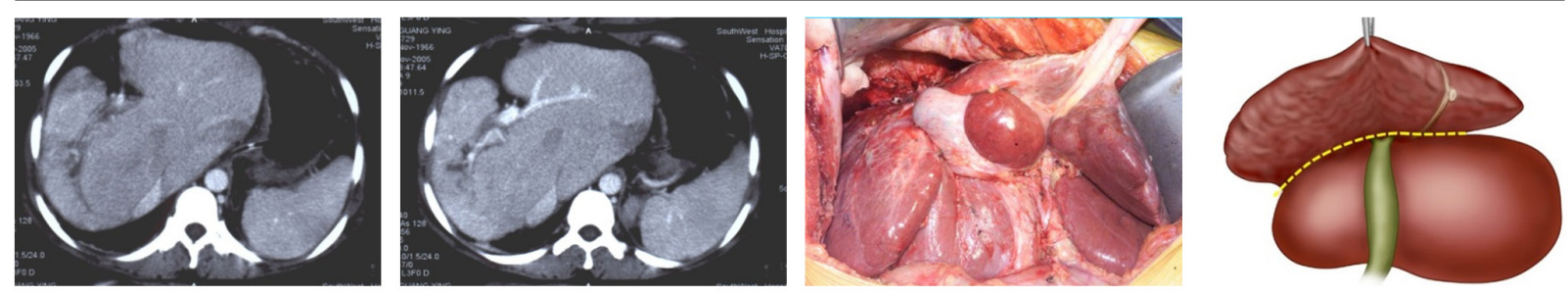

B
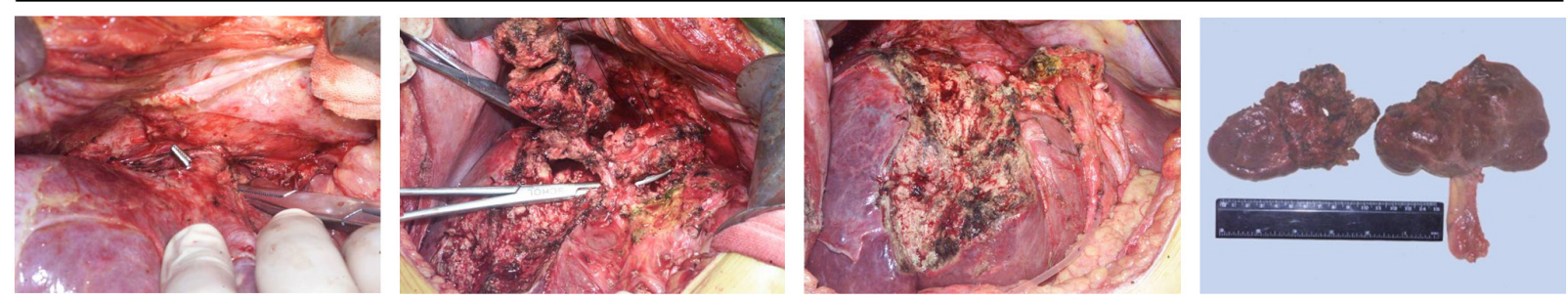

Figure 5. A specific form of bilateral lobectomy excluding the caudate lobe to treat hepatolithiasis. (A), CT images and overall findings; (B), Separation and dissection of liver segments S2-S8, with only segment S1 remaining in the patient.

Table 4. Surgical approaches to treating hepatolithiasis in the literature (2)

\begin{tabular}{|c|c|c|c|}
\hline Series & Years & Primary treatment & No. patients \\
\hline Uchiyama et al. 2007 (16) & $1971-2006$ & Hepatectomy & 38 \\
\hline Otani et al. 1999 (17) & 1980-1996 & Hepatectomy & 26 \\
\hline Cheung \& Kwok 2005 (18) & $1989-2003$ & Hepatectomy & 52 \\
\hline Li et al. $2006(10)$ & $1992-2002$ & Hepatectomy & 161 \\
\hline Nuzzo et al. $2008(8)$ & $1992-2005$ & Hepatectomy & 34 \\
\hline Cheung et al. 2003 (19) & $1993-2001$ & Percutaneous transhepatic cholangioscopic lithotomy or lithotripsy & 79 \\
\hline Li et al. 2005 (20) & $1994-2003$ & Hepatectomy & 46 \\
\hline Kim et al. $2006(21)$ & 1994-2004 & Hepatectomy & 128 \\
\hline Lee et al. 2007 (22) & $2000-2005$ & Hepatectomy & 123 \\
\hline Uenishi et al. 2009 (23) & $1980-2007$ & Hepatectomy & 87 \\
\hline Dong JH et al. & $1975-2008$ & Hepatectomy & 1,175 \\
\hline
\end{tabular}

hepatolithiasis, but the same is not true for East Asia. Moreover, immigration from the Asian-Pacific region means that this rare but emerging disease will pose a therapeutic challenge to doctors in the West as well.

The optimal management of complex hepatolithiasis remains a very difficult and challenging task for hepatobiliary surgeons. Surgery should be indicated for all cases of hepatolithiasis. The current report describes the largest number of patients with hepatolithiasis treated with a hepatectomy (Table 4) and a novel classification to help in determining surgical strategies. Segmentectomy is an effective treatment for type I and type IIb hepatolithiasis. The caudate lobe is a unique segment that is anatomically separate from the rest of the liver; if the liver were a car, the caudate lobe would be its "spare tire". Finally, better selection of which patients should undergo a hepatectomy will lead to better mid-to long-term outcomes.

\section{Acknowledgements}

This project was supported by the Natural Science
Foundation of CQ CSTC, No. 2008BB5134 and Project No. 30801113 of NSFC.

\section{References}

1. Catena M, Aldrighetti L, Finazzi R, Arzu G, Arru $\mathrm{M}$, Pulitanò C, Ferla G. Treatment of non-endemic hepatolithiasis in a Western country. The role of hepatic resection. Ann R Coll Surg Engl. 2006; 88:383-389.

2. Sakpal SV, Babel N, Chamberlain RS. Surgical management of hepatolithiasis. HPB (Oxford). 2009; 11:194-202.

3. Kayhan B, Akdoğan M, Parlak E, Ozarslan E, Sahin B. Hepatolithiasis: A Turkey experience. Turk J Gastroenterol. 2007; 18:28-32.

4. Park HS, Lee JM, Kim SH, Jeong JY, Kim YJ, Lee KH, Choi SH, Han JK, Choi BI. CT Differentiation of cholangiocarcinoma from periductal fibrosis in patients with hepatolithiasis. AJR Am J Roentgenol. 2006; 187:445-453.

5. Mori T, Sugiyama M, Atomi Y. Gallstone disease: Management of intrahepatic stones. Best Pract Res Clin Gastroenterol. 2006; 20:1117-1137.

6. Pockros PJ. Natural progression of untreated 
hepatolithiasis. J Clin Gastroenterol. 2001; 33:95-96.

7. Al-Sukhni W, Gallinger S, Pratzer A, Wei A, Ho CS, Kortan P, Taylor BR, Grant DR, McGilvray I, Cattral MS, Langer B, Greig PD. Recurrent pyogenic cholangitis with hepatolithiasis - The role of surgical therapy in North America. J Gastrointest Surg. 2008; 12:496-503.

8. Nuzzo G, Clemente G, Giovannini I, De Rose AM, Vellone M, Sarno G, Marchi D, Giuliante F. Liver resection for primary intrahepatic stones: A single-center experience. Arch Surg. 2008; 143:570-573; discussion 574.

9. Huang CC. Partial resection of the liver in treatment of intrahepatic stones. Chin Med J. 1959; 79:40-45.

10. Li SQ, Liang LJ, Peng BG, Lai JM, Lu MD, Li DM. Hepaticojejunostomy for hepatolithiasis: A critical appraisal. World J Gastroenterol. 2006; 12:4170-4174.

11. Kusano T, Isa TT, Muto Y, Otsubo M, Yasaka T, Furukawa M. Long-term results of hepaticojejunostomy for hepatolithiasis. Am Surg. 2001; 67:442-446.

12. Leung JW, Yu AS. Hepatolithiasis and biliary parasites. Baillieres Clin Gastroenterol. 1997; 11:681-706.

13. Saing H, Chan KL, Mya GH, Cheng W, Fan ST, Chan FL. Cutaneous stoma in the roux limb of hepaticojejunostomy (hepaticocutaneous jejunostomy): Useful access for intrahepatic stone extraction. J Pediatr Surg. 1996; 31:247-250.

14. Fan ST, Mok F, Zheng SS, Lai EC, Lo CM, Wong J. Appraisal of hepaticocutaneous jejunostomy in the management of hepatolithiasis. Am J Surg. 1993; 165:332-335.

15. Fan ST, Choi TK, Lo CM, Mok FP, Lai EC, Wong J. Treatment of hepatolithiasis: Improvement of result by a systematic approach. Surgery. 1991; 109:474-480.
16. Uchiyama K, Kawai M, Ueno M, Ozawa S, Tani M, Yamaue $\mathrm{H}$. Reducing residual and recurrent stones by hepatectomy for hepatolithiasis. J Gastrointest Surg. 2007; 11:626-630.

17. Otani K, Shimizu S, Chijiiwa K, Ogawa T, Morisaki T, Sugitani A, Yamaguchi K, Tanaka M. Comparison of treatments for hepatolithiasis: Hepatic resection versus cholangioscopic lithotomy. J Am Coll Surg. 1999; 189:177-182.

18. Cheung MT, Kwok PC. Liver resection for intrahepatic stones. Arch Surg. 2005; 140:993-997.

19. Cheung MT, Wai SH, Kwok PC. Percutaneous transhepatic choledochoscopic removal of intrahepatic stones. Br J Surg. 2003; 90:1409-1415.

20. Li X, Shi L, Wang Y, Tian FZ. Middle and long-term clinical outcomes of patients with regional hepatolithiasis after subcutaneous tunnel and hepatocholangioplasty with utilization of the gallbladder. Hepatobiliary Pancreat Dis Int. 2005; 4:597-599.

21. Kim BW, Wang HJ, Kim WH, Kim MW. Favorable outcomes of hilar duct oriented hepatic resection for high grade Tsunoda type hepatolithiasis. World J Gastroenterol. 2006; 12:431-436.

22. Lee TY, Chen YL, Chang HC, Chan CP, Kuo SJ. Outcomes of hepatectomy for hepatolithiasis. World $\mathrm{J}$ Surg. 2007; 31:479-482.

23. Uenishi T, Hamba H, Takemura S, Oba K, Ogawa M, Yamamoto T, Tanaka S, Kubo S. Outcomes of hepatic resection for hepatolithiasis. Am J Surg. 2009; 198:199202.

(Received September 30, 2012; Revised November 3, 2012; Accepted November 9, 2012) 